\title{
Implementation of SRRC Filter in Mobile WiMax with DWT Based OFDM System
}

\author{
Harpreet Kaur \\ DAVIET, Jalandhar, India \\ E-mail: \{harpreet_daviet@yahoo.in\} \\ Manoj Kumar \\ DAVIET, Jalandhar, India \\ E-mail: \{drmanojkumarindia@gmail.co\} \\ Ajay K. Sharma \\ National Institute of Technology, Delhi, India \\ E-mail: \{sharmaajayk@ rediffmail.com\} \\ Harjit P. Singh \\ CTIEMT, Jalandhar, India \\ E-mail: \{harjit_nit@yahoo.co.in\}
}

\begin{abstract}
Pulse shaping filter when applied to the symbols generated for transmission in a bandwidth limited systems allow for bandwidth containment that reduces the Inter-Symbol Interference (ISI) arising from multipath signal reflections which further minimizes the probability of errors at the receiver. Wavelets encompass the advantages of transforming and mapping data onto orthogonal subcarriers with optimal flexibility as well as less complexity which leads to increase the bandwidth efficiency of an Orthogonal Frequency Division Multiplexing (OFDM) system. In this paper Square Root Raised Cosine (SRRC) a pulse shaping technique when applied to Discrete Wavelet Transform (DWT) based OFDM system in mobile WiMax is simulated and its performance analysis in terms of Bit Error rate (BER) as a function of Signal to Noise Ratio (SNR) is investigated for various modulation schemes under AWGN channel. The results obtained show that SRRC filter offers to significantly improve BER for the given SNR and enhance the performance of physical layer in Mobile WiMax. The performance measurements and analysis is done in the simulation developed in MATLAB.
\end{abstract}

Index Terms-DWT-OFDM, pulse shaping, oversampling rate, roll-off factor, Root Raised Cosine, mobile WiMax.

\section{INTRODUCTION}

Mobile Worldwide Interoperability for Microwave Access (WiMax) standard IEEE 802.16e adopted OFDM on its physical layer to facilitate high data rate transmission for wide variety of wireless broadband services with better coverage in the frequency range of $10-66 \mathrm{GHz}$ (Line of sight) and $2-11 \mathrm{GHz}$ (Non Line of
Sight) [1]. The IEEE 802.16 develops the technology specification for WiMax standard air interface which includes the definition of both the physical layers and the medium access control layer for the base station and the subscriber station while WiMax Forum which is an organization that ensure and certify compatibility, conformance and interoperability of products defines its access network operability based on IEEE 802.16 standards [2]. The IEEE 802.16 WiMax standard supports flexibility of its physical layer with multiple physical specifications which facilitates the system designers to modify their system according to their requirements. The physical layer enumerates some mandatory features to be implemented with the system and also includes some optional features to provide a dependable end-to-end link. Conventional Fast Fourier Transform (FFT) based OFDM system in the physical layer of WiMax uses 128 bits to 2,048 point in for mobile communication [3]. However the performance of communication system with OFDM networks using conventional FFT suffers from certain limitations which include sensitivity to Inter-Carrier Interference (ICI), higher ISI, phase noise and band-limited channel which leads to high Peak to Average Power Ratio (PAPR) [5]. Mostly work on orthogonal properties of wavelet in multicarrier modulation system has been exploited to reduce PAPR $[6,7]$. It is also established that in wavelet based OFDM signal, the concept of guard band as Cyclic Prefix $(\mathrm{CP})$ is not required in between every orthogonal signal consequently its bandwidth efficiency was found to increase by approximately $25-30 \%$ [8]. To further improve the performance of digital communication systems based on OFDM systems spectrally efficient filter pulses are employed in practical systems in order to satisfy the bandwidth limitations imposed by the multipath fading channels. Different types of pulse 
shaping filters were applied to the modulated signal to transmit data with reduced ISI and within a limited channel bandwidth [9]. But to have minimum ISI the overall response of transmit filter, receive filter and channel response has to satisfy Nyquist ISI criterion. The most popular pulse shaping filter satisfying Nyquist ISI criterion is the Root Raised Cosine (RRC) pulse [10].

\section{RELATED WORK}

The performance of DWT amid its capability to reduce the loss of orthogonality between the carriers is much better than FFT in OFDM system has been demonstrated with different wavelets in [11]. Kaizuran et al [12] calculated the computational complexity of orthogonal wavelet by exploring its orthonormal base and construction properties and found it to be $O(N)$ which was much less than that of FFT with computational complexity as $\mathrm{O}\left(\mathrm{Nlog}_{2} \mathrm{~N}\right)$. The performance of the Wavelet Packet Modulation (WPM) was observed to perform much better than conventional OFDM for the signals passing through a fading channel and nonlinear High Power Amplifier in [13]. Saad et al in [14] evaluated similar results for the input signal in the presence of Gaussian and Impulsive Noise. Kumbasar et al [15] observed that WPM offered much lower side lobes in transmitted signal thus decreases narrowband Interference and ICI for several multipath wireless channels. Srikanth et al [16] proposed an adaptive loading technique to mitigate ISI when OFDM is used in Wireless Local Area (WLAN). Gandhi et al [17] analyzed that by applying pulse shaping filter to OFDM system increases the width of main lobe to which the peak power is associated and reduces the amplitude of side lobes with which ICI power is associated which results in improvement of the BER performance of OFDM system. The impact of different pulse shaping filters on the OFDM-based WLAN system standard IEEE 802.11a was studied by [18] and Root Raised Cosine (RRC) matched filters was suggested to improve performance in practical WLAN system. A novel filter approach for reducing undesired ICI caused with carrier frequency offset to improve performance was proposed by Mourad et al [19]. SRRC which is a variant of RRC filter, is commonly used as transmit and receive filter in a digital communication system to perform matched filtering. It produces a frequency response with unity gain at low frequencies and complete at higher frequencies, therefore, attenuates the starting and ending portions of the symbol period. SRRC pulses have been proposed as a transmit and receive filters in WiMax (IEEE 802.16 standard), Wideband Code Division Multiple Access (WCDMA) system and 3GPP Long Term Evolution (LTE) in [20].

In this paper, physical layer performance for DWT based OFDM system in mobile WiMax system is analyzed by employing matched SRRC pulse shaping filter in its transmitter and receiver. The assessment of this proposed system with and without pulse shaping filter is demonstrated by means of diverse modulation schemes under AWGN channel. Moreover different aspects of SRRC filter used in its designing are also examined by varying its parameters.

\section{SRRC PULSE SHAPING FILTER}

SRRC filter is applied in both transmitter and receiver in practical DWT based OFDM systems in mobile WiMax so that their combination results in minimum ISI etc. The impulse response of the SRRC filter is given in the following equations (1-3) according to 3GPP standard [20].

$$
p(t)=\frac{\sin \left(\left(\pi \frac{\mathrm{t}}{\mathrm{T}}(1-\alpha)\right)+4 \alpha \frac{\mathrm{t}}{\mathrm{T}} \cos \left(\left(\pi \frac{\mathrm{t}}{\mathrm{T}}(1+\alpha)\right)\right.\right.}{\pi \frac{\mathrm{t}}{\mathrm{T}}\left(1-\left(4 \alpha \frac{\mathrm{t}}{\mathrm{T}}\right)^{2}\right)}
$$

with following values at

(i) $\mathrm{t}=0$

$$
p(t)=(1-\alpha)+\frac{4 \alpha}{\pi}
$$

(ii) at $\frac{t}{T}=\frac{1}{4 \alpha}$

$$
p(t)=\frac{\alpha}{\sqrt{2}}\left(\left(1+\frac{2}{\pi}\right) \sin \left(\frac{\pi}{4 \alpha}\right)+\left(1-\frac{2}{\pi}\right) \cos \left(\frac{\pi}{4 \alpha}\right)\right)
$$

where $\alpha$ is the roll-off factor and T is the symbol duration.

The frequency response of the SRRC filter is mainly characterized by following factors such as:-

a) The roll-off factor $(\alpha)$ determines the sharpness of the frequency response as it governs the bandwidth occupied by the pulse and the rate at which the tails of the pulse decay. It lies in the range 0 to 1 with value $\alpha=0$ offers the narrowest bandwidth while decay in time domain is fastest, but with $\alpha=1$ the spectrum requires twice as much bandwidth and the slowest rate of decay in the time domain. For most practical digital communications systems, roll-off factor between 0.2 and 0.5 has proved to be very proficient with adequate bandwidth and smaller tails contributing to less ISI [21].

b) For a signal to be sent through a digital filter another parameter is the oversampling factor ( $\eta$ ). The oversampled signal by a factor of $\mathrm{N}$ means it is sampled at $\mathrm{N}$ times the Nyquist rate. Oversampling a signal aids to improves resolution, reduces noise and helps avoiding aliasing and phase distortion but the main drawbacks to this approach is that the processor must handle this higher data rate while performing additional processing on the data to implement the digital filter [21]. 


\section{PRoposed ModeL}

The proposed model has been designed to schematically evaluate the physical layer performance of DWT based OFDM system in the mobile WiMax with SRRC pulse shaping filter applied in its transmitter and receiver which is further examined by varying its parameters such as roll-off factor and oversampling factor for various modulation schemes such as 16QAM and 64QAM under AWGN channel. The flow chart designed for the model is presented with transmitter and receiver modules as given in figure 1(a) and 1(b) respectively.

\section{(i) Transmitter}

The basic steps in derivation of the transmitter in the proposed system are as follows:

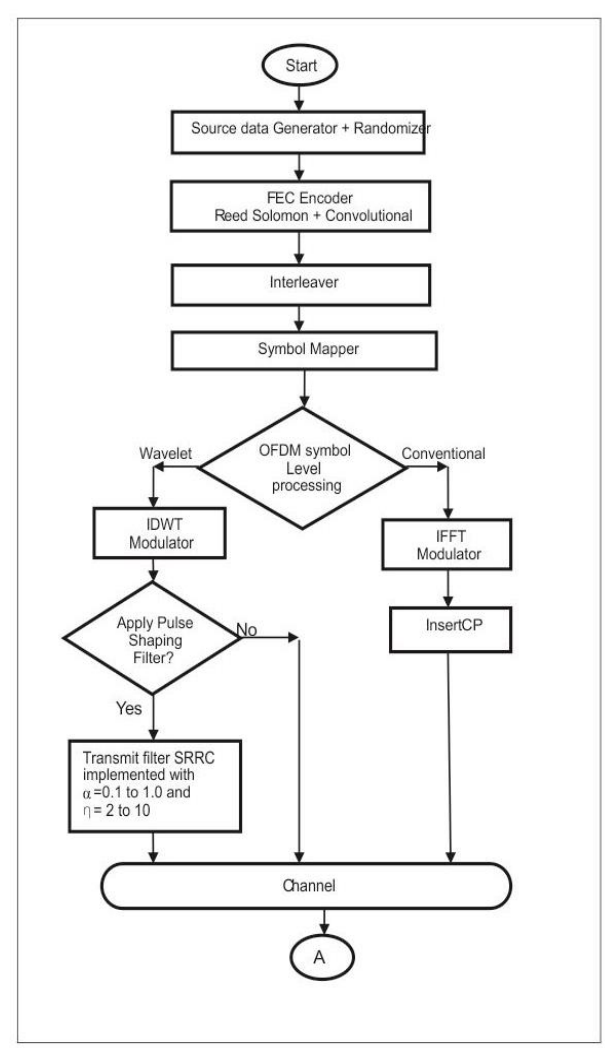

Fig.1(a). Flow Chart of the Transmitter in the Proposed System.

Step 1: Source data is generated in the form of digital random numbers and the length of its information bits (x) depends upon the type of the modulation scheme (16QAM or 64QAM) used to map the bits to symbols.

Step 2: The randomized data is fed to the ReedSolomon (RS) encoder which is a part of Forward Error Correction (FEC). This RS code is represented in the form as RS (p, q, s), where $\mathrm{p}$ is total number of data and parity symbols, $\mathrm{q}$ is the number of input data symbols to be encoded, and $\mathrm{s}$ is the maximum number of data symbols that can be corrected. For $\mathrm{x}$ as the number of bits per symbol, the value of $\mathrm{p}$ and $\mathrm{q}$ is calculated using equation

$$
\begin{gathered}
\mathrm{p}=2^{\mathrm{x}}-1 \\
\mathrm{q}=2^{\mathrm{x}}-1-2 \mathrm{~s}
\end{gathered}
$$

RS error-coded bits are further coded by Convolutional (CC) encoder; here each $r$ bit symbol is transformed into $\mathrm{k}$ bit symbol to have $\mathrm{r} / \mathrm{k}$ as their code rate. This transformation of $\mathrm{r}$ bit symbol into $\mathrm{k}$ bit symbol depends upon the last $\mathrm{m}$ data symbols, where $\mathrm{m}$ is the constraint length of the CC code. Coding Rates for different modulation schemes used in this simulation is shown in table 1 .

Table 1. Code Rates for Different Modulation Schemes

\begin{tabular}{|c|c|c|c|c|}
\hline $\begin{array}{c}\text { Sr } \\
\text { no }\end{array}$ & Modulation & RS code & $\begin{array}{c}\text { CC code } \\
\text { rate }\end{array}$ & $\begin{array}{c}\text { Overall } \\
\text { code rate }\end{array}$ \\
\hline 1. & $16 \mathrm{QAM}$ & $(64,48,4)$ & $2 / 3$ & $1 / 2$ \\
\hline 2. & $16 \mathrm{QAM}$ & $(80,72,4)$ & $5 / 6$ & $3 / 4$ \\
\hline 3. & 64QAM & $(108,96,6)$ & $3 / 4$ & $2 / 3$ \\
\hline 4. & 64QAM & $120,108,6)$ & $5 / 6$ & $3 / 4$ \\
\hline
\end{tabular}

Step 3: To protect the data further from burst errors during transmission, this FEC encoded data is then fed into a block Interleaver.

Step 4: The incoming (interleaved) bits is mapped onto different sub-carriers by the symbol mapper in this step, where depending upon its size the data is modulated using different modulation schemes.

Step 5: In the next step OFDM waveform for WiMax physical layer is created by OFDM modulator that basically gives sub-carriers its orthogonality and transforms its frequency spectrum into a time domain signal which can be implemented as:-

Case 1: Conventional OFDM implementation in the physical layer of mobile WiMax uses IFFT during transmission, wherein to mitigate ISI effect $\mathrm{CP}$ as a guard interval period is added to the signal to provide a protective separation between transmitted OFDM frames.

Case 2: In Wavelet based OFDM implementation Inverse Discrete Wavelet Transform (IDWT) modulator is employed which doesn't require the use of CP hence decreases the bandwidth wastage and also its transmission power.

Step 6: For the DWT- OFDM system employing a SRRC filter in its transmitter following system parameters are set in this step to design the filter where (i) $F_{s}$ is the sampling frequency (ii) $F_{c}$ is the cutoff frequency (iii) $T_{p}$ is the type specification of filter, it is SRRC filter in this model (iv) $\alpha$ as the roll-off factor (v) $\eta$ as oversampling rate (v) $d$ as delay and (vi) $F_{d}$ as filter order. System parameters values of SRRC filter is given in table 2 and is implemented utilizing equations (1-3) is the MATLAB command. 
Step 7: This proposed model incorporate AWGN for channel implementation.

\section{(ii) Receiver}

The receiver performs the reverse operation of the transmitter. Various steps in derivation of the receiver in this proposed model are as follows:

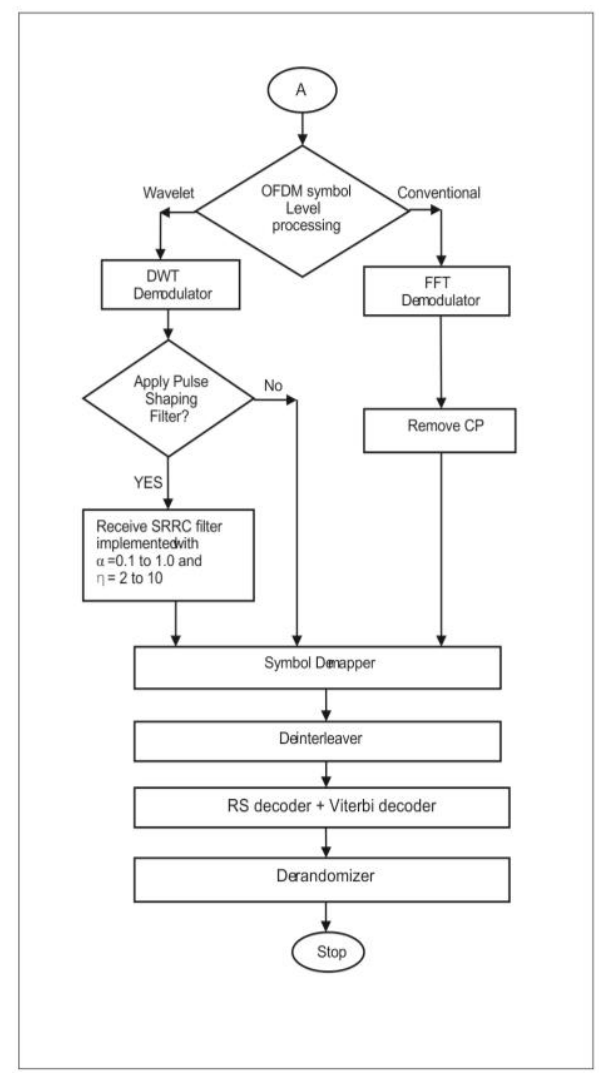

Fig.1(b). Flow Chart of the Receiver in the Proposed System.

Step 1: OFDM demodulation involves conversion of signal from time domain to frequency domain which is achieved as:-

Case 1: In conventional case FFT demodulator is implemented, and then $\mathrm{CP}$ is removed before the signal is passed through various other blocks of the receiver.

Case 2: In wavelet based OFDM, DWT demodulator is employed to perform this conversion. Further for the case where SRRC filter is employed in the transmitter matched SRRC filter as mentioned in the equations (1-3) is applied in the receiver as well to minimize ISI effects.

Step 2: In this step demapper at the receiver demodulated symbols to generate data bit stream corresponding to the modulation and symbol mapping in the transmitter.

Step 3: The next step is the deinterleaving operation which rearranges the bits into original form.

Step 4: Deinterleaved data is then fed to FEC decoder having RS decoder and Viterbi decoder for decoding.

Step 5: The decoded data is finally derandomized to obtain the output data.
Finally, the comparison of BER is done for different cases at different SNRs.

\section{RESULTS AND ANALYSIS}

The physical layer performance of DWT based OFDM system in mobile WiMax system with SRRC filter under AWGN channel is measured in BER curves for different modulation techniques such as 16QAM $1 \frac{1}{2}, 16 \mathrm{QAM}^{3} / 4$, 64QAM $2 / 3$ and 64QAM $3 / 4$ modulation schemes.

Table 2. SRRC Filter Specifications and Mobile WiMax Parameters

\begin{tabular}{|c|c|}
\hline Parameters & Value \\
\hline Sampling frequency & $11.2 \mathrm{MHz}$ \\
\hline Roll-off factor & 0.1 to 1.0 \\
\hline Oversampling Rate & 2 to 10 \\
\hline SNR Value & 0 to 35 dB \\
\hline Channel Encoding & $\begin{array}{c}\text { Reed-Solomon Encoder }+ \\
\text { Convolution Encoder }\end{array}$ \\
\hline Decoder & $\begin{array}{c}\text { RS decoder }+ \\
\text { Viterbi decoder }\end{array}$ \\
\hline Modulation Scheme & 16 QAM $1 / 2,16$ QAM $3 / 4$, \\
& QAM $2 / 3$ and 64 QAM $3 / 4$ \\
\hline Channel model & AWGN \\
\hline Noise & 1000 \\
\hline No. of Symbols & $1024+1 / 4$ \\
\hline FFT size + Cyclic Prefix & Bior5.5 \\
\hline DWT Family &
\end{tabular}

In particular, the performance of different parameters of SRRC filter such as the oversampling rate and roll-off factor are evaluated and compared. Simulation is implemented in MATLAB. Different code rate used for modulation techniques is given in table 1. Mobile WiMax parameters and the specifications of SRRC filter used in the simulation are shown in table 2 .

Case -I: Impact of oversampling rate (parameter of an SRRC filter) varying from 2 to 10 on the performance of DWT based OFDM system for various modulation schemes.

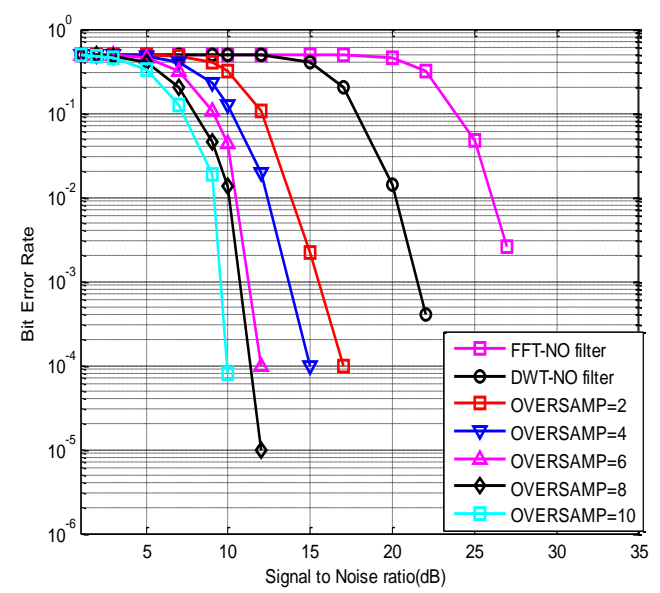

(a) 


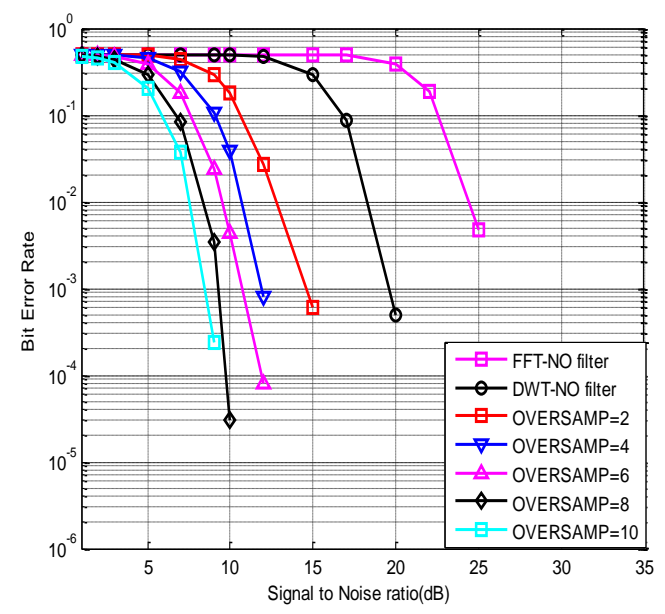

(b)

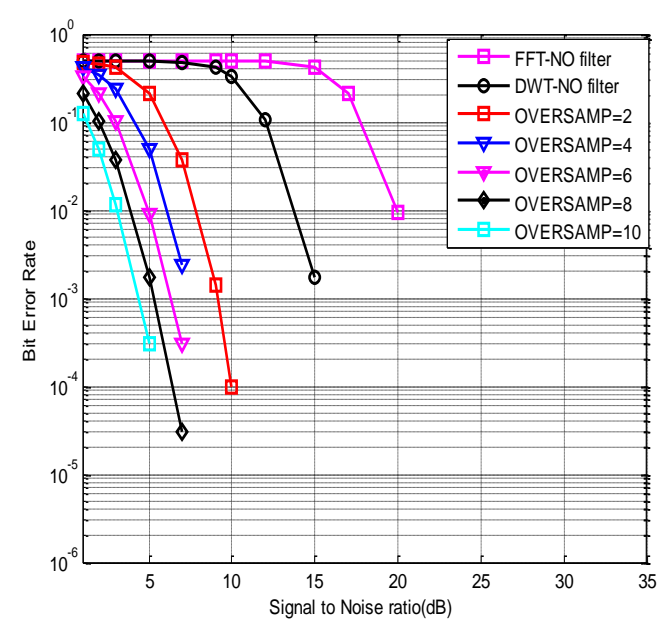

(c)

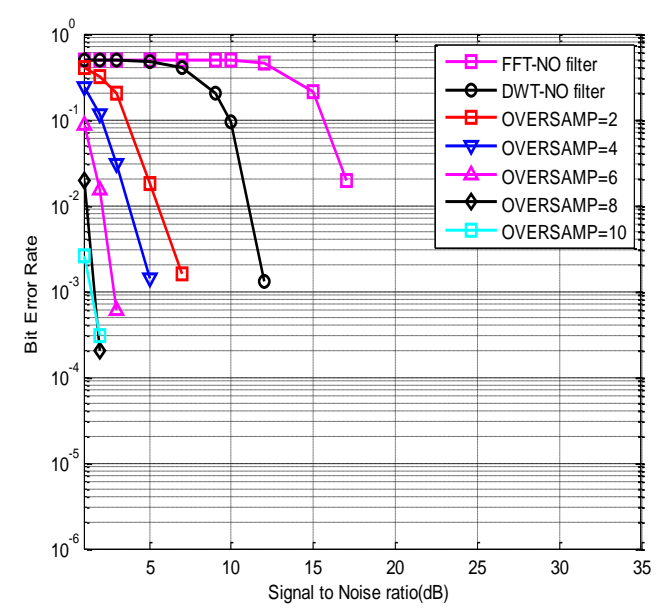

(d)

Fig.2. Performance Comparison of DWT-OFDM System with SRRC Filter for Oversampling Rate Varying from 2 to 10 for (a) 64QAM 3/4 (b) 64QAM $2 / 3$ (c) 16QAM $3 / 4$ and (d) 16QAM $1 / 2$ Modulation Scheme.

Fig. 2 clearly illustrates that physical layer performance of DWT based OFDM system is significantly improved in comparison with conventional FFT based OFDM system in mobile WiMax for modulation scheme 64QAM $3 / 4,64 \mathrm{QAM}^{2} / 3$, and 16QAM $3 / 4$ and 16QAM $1 \frac{1}{2}$ with AWGN channel. Further, comparative performance analysis of DWT based OFDM system by employing SRRC pulse filter with a different oversampling rate $(\eta)$ such as 2, 4, 6, 8 and 10 is measured in BER curves. From Fig. 2(a), it is observed that the SRRC pulse with $\eta=8$ significantly enhance the performance with minimum BER obtained as $10^{-5}$ at SNR equals to $12 \mathrm{~dB}$ for modulation scheme 64QAM $3 / 4$. In Fig. 2(b) and 2(c), BER as low as $10^{-4}$ is obtained for $\eta=8$ at SNR equals to $12 \mathrm{~dB}$ for modulation technique 64QAM $2 / 3$ and at SNR equals to $7 \mathrm{~dB}$ with the modulation scheme 16QAM $3 / 4$ respectively. Fig. 2(d) shows that minimum BER of $10^{-4} \mathrm{~dB}$ with $\eta=8$ is observed at SNR equals to $2 \mathrm{~dB}$ for modulation schemes 16QAM $1 / 2$. It is apparent from the results obtained that employing SRRC filter in the physical layer of the DWT based OFDM system of mobile WiMax significantly enhances its performance which varies with different oversampling rate (n) and the best results are with oversampling rate of 8 for different modulation schemes and minimum BER is observed in 64QAM $3 / 4$ modulation schemes under AWGN channel. The result obtained also revealed that performance is observed to be better at high values of SNRs with higher order modulation scheme while lower order schemes perform better for lower SNRs.

Case -II: Impact of roll-off factor (parameter of an SRRC filter) varying from 0.1 to 0.75 on the performance of DWT based OFDM system for various modulation schemes.

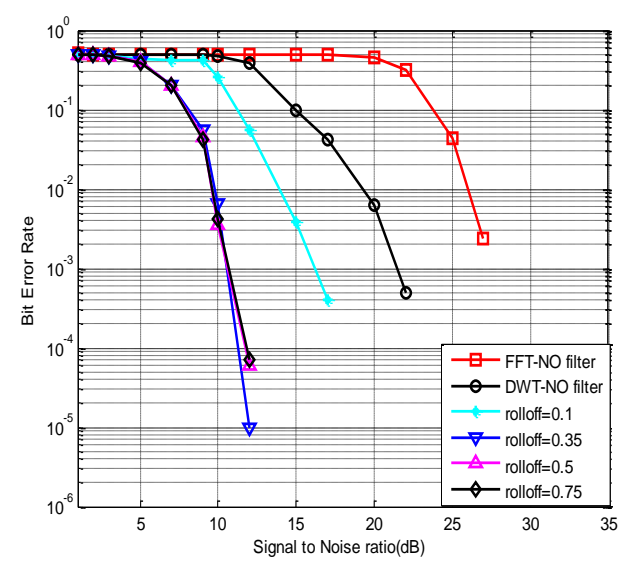

(a)

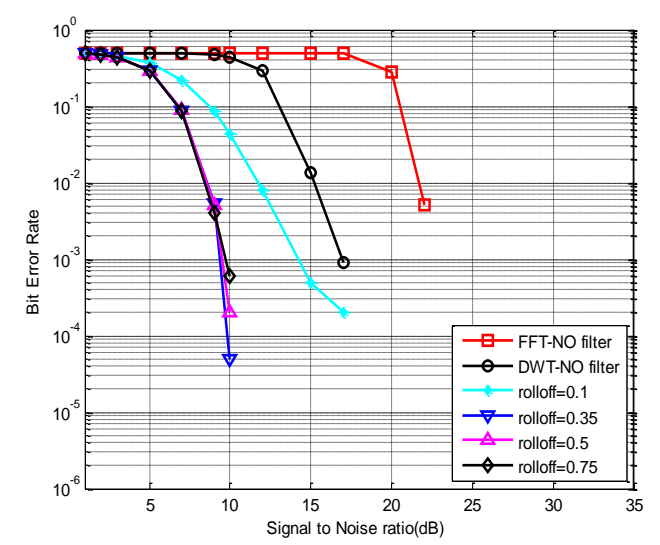

(b) 


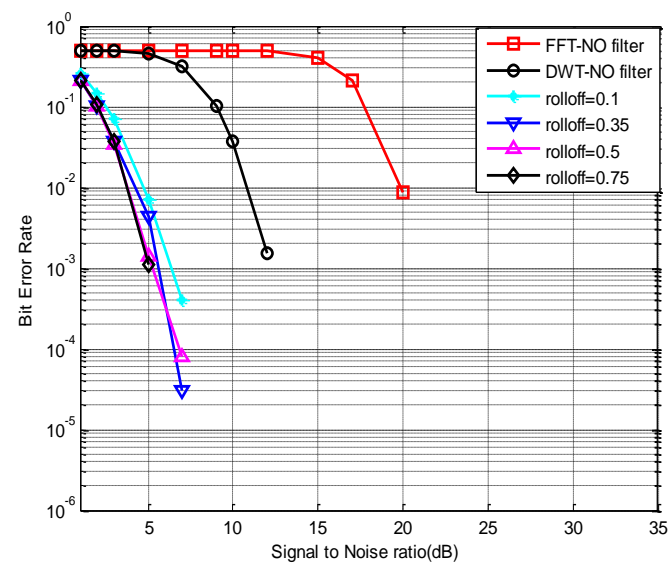

(c)

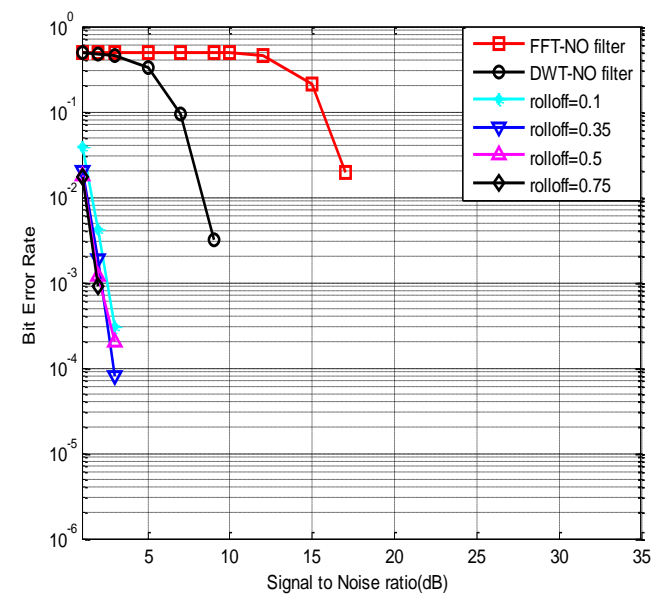

(d)

Fig.3. Performance Comparison of DWT-OFDM System with SRRC Filter for roll-off Factor Varying from 0.1 to 0.75 for (a) 64QAM $3 / 4$ (b) 64QAM $2 / 3$ (c) 16QAM $3 / 4$ and (d) 16QAM $1 \frac{1}{2}$ Modulation Scheme.

Fig.3 shows that by employing SRRC filter in DWT based OFDM system, dependence of BER on the roll-off factor $(\alpha)$ which is varied from 0.1 to 0.75 is observed for different modulation schemes under AWGN channel. As illustrated in the Fig. 3(a), for the modulation scheme 64QAM $3 / 4$, SRRC pulse with $\alpha=0.35$ exhibits much better performance in comparison with other roll-off factors with the value of BER obtained as $10^{-5}$ at $12 \mathrm{~dB}$. In Fig. 3(b) and 3(c), SRRC pulse with $\alpha=0.35$ exhibit BER as low as $10^{-4}$ for the SNR value of $10 \mathrm{~dB}$ and $7 \mathrm{~dB}$ for the modulation scheme 64QAM $2 / 3$ and $16 \mathrm{QAM}^{3} / 4$ respectively. Fig. 3(d) shows that for modulation schemes 16QAM $1 / 2$, SRRC filter with $\alpha=0.35$ with BER value of $10^{-4}$ at SNR equals to $3 \mathrm{~dB}$ is marginally better than with $\alpha=0.5$. An improvement of $\sim 5 \mathrm{~dB}$ is observed in every case with the introduction of SRRC filter in the physical layer of the WiMax system. Thus it is inferred that with SRRC filter in the physical layer of DWT based OFDM system of mobile WiMax reduces the BER with most optimal value of roll-off factor found as 0.35 and hence improves the performance with 16QAM and 64QAM modulation techniques.

\section{CONCLUSION}

In the present work, physical layer performance of mobile WiMax IEEE $802.16 \mathrm{e}$ is simulated and tested by implementing of SRRC matched filters in transmitter and receiver system of the wavelet-based OFDM system of mobile WiMax system. The results obtained revealed significant improvement for various QAM modulation techniques in AWGN channel. The investigations are carried further by analyzing the impact of varying certain parameters such as the roll-off factor and oversampling rate on the performance of the proposed model and the best results were found with oversampling factor equals to 8 for 64QAM $3 / 4$ and with roll-off factor value equals to 0.35 for 64QAM and 16QAM modulation scheme. This study will be useful to achieved much lower bit error rates and can be used for high transmission rates in mobile WiMax with the possibility of implementation of SRRC filter by assuming the reasonable choice of its parameters. In future, the same scheme can be implemented for multiple inputs multiple output systems and in a multiuser environment.

\section{REFERENCES}

[1] IEEE Std 802.16e TM-2005, "Part 16: Air interface for fixed and mobile broadband wireless access systems," Feb. 2006.

[2] Etemad, Kamran. "Overview of mobile WiMax technology and evolution."Communications magazine, IEEE 46, no. 10 pp.31-40, 2008.

[3] S. Casal, Pedro, J. R. Pineiro, J.A. G.Naya, and L. Castedo. "Experimental evaluation of the WiMax downlink physical layer in high-mobility scenarios." EURASIP Journal on Wireless Communications and Networking, vol 2015, article no. 21, 2015.

[4] Y.Li and G.L Stuber, "Orthogonal Frequency Division Multiplexing for Wireless Communication," Springer; 2006.

[5] P. Thieny, V. B. Mark and M. Marc, "BER Sensitivity of OFDM Systems to Carrier Frequency Offset and Wiener Phase Noise," IEEE Transaction on Communication, vol. 43, pp 191-193, 1995.

[6] S. Khalid, and S. I. Shah, "PAPR Reduction by using discrete wavelet transform," in Proceeding of IEEE $-2^{\text {nd }}$ International Conference on Emerging Technologies, pp 179-182, 2006.

[7] J. Armstrong, "Analysis of new and existing methods of reducing Inter-Carrier Interference due to carrier frequency offset in OFDM," IEEE Transaction on Communication, vol. 47,no. 3, pp 365-369, 1999.

[8] M. K. Gupta and S. Tiwari, "Performance evaluation of conventional and wavelet based OFDM System," International Journal on Electronics and Communication, vol. 67, no.4, pp 348-354, 2013.

[9] Li Xiaodong and L.J. Cimini, “.Effects of clipping and filtering on the performance of OFDM", IEEE communications Letters, vol. 2 no.5, pp131-133, 1998.

[10] P. Tan and N.C. Beaulieu, "Reduced ICI in OFDM systems using the better than raised-cosine pulse", IEEE Communication Letter, vol. 8, no. 3, pp 135-137, 2004.

[11] H. Kaur, M. Kumar, A. K Sharma and H.P.Singh, "Performance Analysis of Different Wavelet Families over Fading Environments for Mobile WiMax System," 
International Journal of Future Generation Communication and Networking, vol. 8, pp 87-98, 2015.

[12] A. Kaizuran and Z. M. Hussain "Studies on DWT-OFDM and FFT-OFDM Systems", in the Proceeding of IEEE International Conference on Communication, Computer and Power, pp 382-386, 2009.

[13] B. G. Negash and H. Nikookar, "Wavelet-based multicarrier transmission over multipath wireless channels," Electronics Letters, vol. 36, no. 21, pp 1787$1788,2000$.

[14] W.Saad, N.El-Fishawy, S.EL-Rabaie and M. Shokair, "An Efficient Technique for OFDM system using Discrete Wavelet Transform," Springer-Verlag Berlin Heidelberg, pp 533-541, 2010.

[15] V.Kumbasar, and O. Kucur, "Performance comparison of wavelet based and conventional OFDM systems in multipath Rayleigh fading channels," Digital Signal Processing, vol. 22, pp 841-846, 2012.

[16] S. Srikanth, V. Paul Reuben, P. Arun, S. Thiagarajan, "Performance of adaptive modulation techniques for wireless local area networks," IETE Technical Review, Special Issue on Broadband Wireless Technologies, vol. 21, pp 345-350, 2004.

[17] D. Gandhi, S. Gupta and U. D. Dalal, "Article: Implementation of Pulse Shaping Techniques in OFDM System," International Journal of Computer Applications, vol. 68, no.10, pp 19-23, 2013.

[18] P. Xiao, C. Toal, D. Burns, V. Fusco and C. Cowan, "Transmit and receive filter design for OFDM-based WLAN systems," in the proceeding of International Conference on Wireless Communications and Signal Processing, 2010.

[19] H.M. Mourad "Reducing ICI in OFDM systems using a proposed pulse shape," Wireless Personnel Communication, vol. 40, pp 41-48, 2006.

[20] L. Korowajczuk, "LTE, WiMax and WLAN Network Design, Optimization and Performance Analysis," John Wiley and Sons Ltd Publishers, 2011.

[21] K. Vaisanen and M Renfors "Efficient digital filters for pulse-shaping and jitter-free frequency error detection and timing recovery", Signal Processing, vol.81, pp 829-844, 2001.

\section{Authors' Profiles}

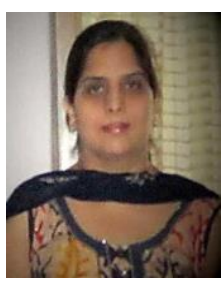

Harpreet Kaur received her B.Tech degree in Electronics and Communication Engineering in 1996 from Guru Nanak Dev University Amritsar. Completed M.E degree in Computer Science \& Engineering from NITTTR under Panjab University, Chandigarh in 2004. She is presently working as Assistant Professor in the Department of Computer Science \& Engineering in DAV Institute of Engineering \& Technology, Jalandhar. She is also a research scholar for Ph.D. in Computer Science and Engineering in Punjab Technical University, Jalandhar and pursuing her research in wireless networks. Her research interests include wireless communication, networking and signal processing.

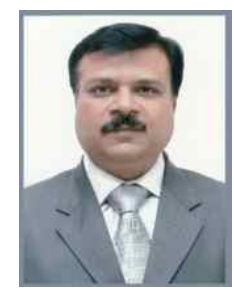

Manoj Kumar received his B.E (ECE) from Gulbarga University in the year 1990 and M.Tech. (ECE) from Punjab Technical University, Jalandhar in the year 2001. He completed his Ph.D. from Punjab Technical University, Jalandhar in the year 2007. From 1991 to 2001, worked as the lecturer and thereafter from July 2001 till July 2010 as Vice-Principal \& Faculty Head in Department of ECE and research centre at DAV Institute of Engineering \& Technology, Jalandhar. He worked in CT Institute of Engineering Management \& Technology (CTIEMT), Jalandhar as the Group Director from July 2010 till August 2015 and presently working as Principal DAVIET, Jalandhar. He has published 62 research papers in the International/National Journals/Conferences, authored 8 Engineering books and reviewed 5 Engineering books. His area of interest is optical fiber communication and wireless communication.

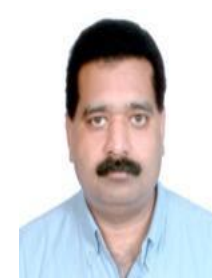

Ajay K. Sharma received his B.E (ECE) degree from Punjab University, Chandigarh in the year 1986, MS in Electronics and Control from Birla Institute of Technology (BITS), Pilani in the year 1994 and Ph.D. in Electronics Communication and Computer Engineering in the year 1999.

After serving various organizations from 1986 to 1995, he joined National Institute of Technology (Erstwhile Regional Engineering College) Jalandhar as Assistant Professor in the Department of Electronics and Communication Engineering in the year 1996. From 2001, he worked as Professor in the ECE department and thereafter he has worked as Professor in Computer Science \& Engineering from 2007 to 2013 in the same institute. He joined as Director, NIT Delhi in 2013 and presently working in NIT Delhi. His major areas of interest are broadband optical wireless communication systems and networks, dispersion compensation, fiber nonlinearities, optical soliton transmission, WDM systems and networks, Radio-over-Fiber (RoF) and wireless sensor networks and computer communication. He has published 272 research papers in the International/National Journals/Conferences and 12 books. He has supervised $18 \mathrm{Ph} . \mathrm{D}$. and 48 M.Tech theses. He has completed two R\&D projects funded by Government of India and one project is ongoing. He is the technical reviewer of reputed international journals likeOptical Engineering, Optics letters, Optics Communication, Digital Signal Processing. He has been appointed as the member of technical Committee on Telecom under International Association of Science and Technology Development (IASTD) Canada for the term 2004-2007.

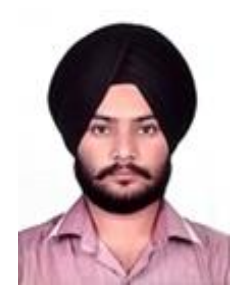

Harjit Pal Singh received his Ph.D. from National Institute of Technology, Jalandhar and joined as Assistant Professor, Department of Electronics and Communication at CT Institute of Engineering, Management and Technology, Jalandhar. His area of interest is Voice over Internet Protocol, wireless communication, signal processing, digital filter design and wireless sensor networks. His research and teaching experience is 7 years. He has published 40 research papers in referred/indexed journals and international conferences. He has attended and presented his research findings in different countries such as France, UK, USA, Dubai, Hong Kong etc. He has guided 3 Ph.D. 
dissertations and guiding more than 12 M.Tech dissertations. $\mathrm{He}$ is Associate Editor and member of Editorial Board of various reputed journals. He is the reviewer of IEEE Network Magazine, Journal of Computer Application and Networks (Elsevier), Circuits, Systems, and Signal Processing (Springer), International Journal of Electronics (Taylor \& Francis),
Mosharaka Journals and Conferences, WSEAS Journals and Conferences, IAENG Journals and Conferences, SDIWC Journals and Conferences. $\mathrm{He}$ is the member of various scientific \& technical societies such as The British Science Association Communication, CSTA/ACM, IAENG, IACSIT, UACEE, IRED, SDIWC, ISAET, IISRO.

How to cite this paper: Harpreet Kaur, Manoj Kumar, Ajay K. Sharma, Harjit P. Singh,"Implementation of SRRC Filter in Mobile WiMax with DWT Based OFDM System", International Journal of Computer Network and Information Security(IJCNIS), Vol.8, No.4, pp.62-69, 2016.DOI: 10.5815/ijcnis.2016.04.08 